\title{
Uso de Facebook en la promoción de hoteles categoría lujo de Manta en tiempo de COVID-19
}

\section{Use of Facebook in the promotion of luxury category hotels in Manta in time of COVID-19}

\author{
Rafael Vera Vera ${ }^{1}$, Fernando Veloz Camejo ${ }^{2 *}$, Yadira Párraga Basurto ${ }^{3}$ \\ ${ }^{1}$ Escuela Superior Politécnica Agropecuaria de Manabí “Manuel Félix López”, Calceta, Manabí, Ecuador. \\ jose_verav@espam.edu.ec \\ https://orcid.org/0000-0003-1721-8770 \\ ${ }^{2}$ Escuela Superior Politécnica Agropecuaria de Manabí “Manuel Félix López”, Calceta, Manabí, Ecuador. \\ 凶ashington.veloz@espam.edu.ec \\ https://orcid.org/0000-0002-7113-2049 \\ ${ }^{3}$ Escuela Superior Politécnica Agropecuaria de Manabí “Manuel Félix López”, Calceta, Manabí, Ecuador. \\ 凶yparraga@espam.edu.ec \\ https://orcid.org/0000-0003-2490-5827
}

\section{Resumen}

La pandemia del COVID-19 ha golpeado fuertemente al sector empresarial en todo el planeta y ha ocasionado un distanciamiento social, obligando a las empresas a potenciar el uso de las redes sociales como una estrategia de supervivencia. Esta investigación buscó evaluar el uso de la red social Facebook para la promoción turística en hoteles categoría lujo, de la ciudad de Manta, durante la pandemia. Para el levantamiento de la información, se realizó una revisión de la interacción entre los hoteles y los usuarios de esta red social mediante el modelo Presencia, Respuesta, Generación y Sugerencia (PRGS) tomando como base los datos obtenidos a través de la plataforma Fanpage Karma. Se evaluó el nivel de engagement por parte de los seguidores y se realizó un análisis cualitativo de los comentarios realizados durante la pandemia mediante el software Atlas.Ti v.9. Se identificaron 9 códigos relacionados con: concurso, en vivo, etiquetado e interacción entre usuarios, oferta de servicios, opinión sobre el hotel y sus servicios, opinión sobre la ciudad, saludos y agradecimientos, solicitud de información, y vinculados directamente a la pandemia. Se concluyó que el nivel de engagement de los seguidores de los hoteles categoría lujo de Manta en la red social Facebook están por debajo de los niveles aceptables.

Palabras clave: COVID-19, Facebook, redes sociales, hoteles.

\begin{abstract}
The COVID-19 pandemic has aggressively hit the business sector worldwide and has caused social distancing, forcing companies to promote the use of social networks as a survival strategy. This research evaluates the use of Facebook for tourism promotion of luxury hotels in the city of Manta during the pandemic. To collect the information, a review of the interaction between the hotels and the users of this social network was carried out through the Presence, Response, Generation and Suggestion (PRGS) model based on the data obtained through the Fanpage Karma platform. The level of engagement by the followers was evaluated and a qualitative analysis of the comments posted during the pandemic was carried out using the Atlas. Ti v.9 software. 9 codes were identified related to: contest, live, labeling and interaction between users, service offer, opinion about the hotel and its services, opinion about the city, greetings and thanks, request for information, and directly linked to the
\end{abstract}


pandemic. It was concluded that the level of engagement of followers of Manta's luxury category hotels in the social network Facebook is below acceptable levels.

Keywords: COVID-19, Facebook, social networks, hotels

\section{Introducción}

El internet se ha constituido -prácticamente- en un derecho social (Miranda Bonilla, 2016). Su progreso y evolución tecnológica ha potenciado el avance de la comunicación, ofreciendo muchas posibilidades de interacción y acceso global a la información (Alcalá Casillas, 2017). El alcance de su difusión, la ha convertido en una importante herramienta de conexión inmediata y actualizada, no solamente en el contexto social, sino también entre la comunidad científica y la sociedad, cuyo flujo de información toma lugar principalmente en la web 2.0 (López-Pérez y Olvera-Lobo, 2016) como efecto de la facilidad que brinda a los usuarios para compartir sus opiniones sobre la información que se difunde en un sitio de visita.

Entre los canales de difusión de información más utilizados en la web 2.0, se encuentran las redes sociales, las cuales posibilitan la interacción con los usuarios, quienes cada vez, de forma anónima, van tomando mayor importancia en la construcción de productos a través de la influencia de sus comentarios, especialmente en el caso hotelero (Ortiz García y González Sánchez, 2014; Parra-Meroño et al, 2017). En este sentido, las redes sociales se han convertido en un potente canal de promoción de productos o servicios hoteleros y, por su capacidad de interacción con los usuarios, en una herramienta para el cuidado de la reputación online (Morán Quiñonez y Cañarte-Rodríguez, 2020). Por esta razón, la presencia activa de los hoteles en estos entornos digitales es sustancial para su éxito en el mercado.

En los últimos 40 años, el mundo ha experimentado un considerable número de epidemias y pandemias, pero ninguna con las implicaciones económicas del Síndrome Agudo Respiratorio Severo SARS-2 conocido mundialmente como el COVID-19 (Gössling et al., 2020) por lo que la lucha por mantener una presencia en el mercado ha sido crucial para la supervivencia de una empresa, y en este espacio las redes sociales resultan de gran utilidad.

Aunque el modo de interacción a través del internet, entre los hoteles y sus clientes venía dando señales de grandes avances, la pandemia del COVID-19, obligó a las empresas a incrementar sus esfuerzos en la gestión de contenidos promocionales a través de la web. En consecuencia, las redes sociales fortalecieron su reputación como plataformas útiles para las empresas en cuanto al contacto e interacción con los clientes y medio de fidelización; en este contexto, el sector hotelero no fue un caso aislado.

En una mirada retrospectiva previo a la pandemia, según Duarte (2019) América Latina (AL) es la región con los usuarios de mayor consumo de tiempo en redes sociales del mundo. Hoy, sin duda el aislamiento social ha incrementado esa realidad. Pese a la extensa variedad de redes sociales, Facebook concentra el mayor número de usuarios en $\mathrm{AL}$, de acuerdo a Burgueño (2020) Ecuador ocupa el séptimo lugar en la lista. La utilidad de esta red social en el contexto de la promoción no es reciente (Altamirano, 2015; Chierichetti, 2012; Mellinas et al., 2014; Sánchez Amboage, 2010; Zazueta Hernández y Olmos Martínez, 2018) sobre todo para atraer turistas internacionales (Hsu, 2012) sin embargo, la efectividad de su marketing es un gran desafío, tanto desde la óptica académica como de las empresas (Leung, 2013).

Hasta julio del 2020, 630 de los 700 grandes y medianos hoteles que agrupa la Federación Hotelera del Ecuador, se mantenían cerrados, ocasionando perjuicios millonarios (El Comercio, 2020) con porcentajes de ocupación no mayor a un $9 \%$, que según los hoteles, ya representan la pérdida de los años 2020 y 2021, por lo que muchos hoteleros han declarado al sector en quiebra (E1 Universo, 2020). Este panorama, no es ajeno al del sector hotelero de la ciudad de Manta, ubicada en la provincia de Manabí, una de las urbes con mayor dinamismo turístico y la de mayor concentración de hoteles de la costa manabita, por su conectividad nacional e internacional, ya que posee un aeropuerto y un puerto marítimo de aguas profundas frecuentemente visitado por cruceros de todo el mundo (Félix et al., 2020).

En el marco de las afectaciones causadas por la pandemia del COVID-19 detectadas en la ciudad de Manta, Félix y García Reinoso (2020) determinaron 
que una de las estrategias de reactivación turística, es la promoción en redes sociales. En este contexto, el propósito de esta investigación es evaluar el uso de Facebook en la promoción de hoteles categoría lujo de Manta durante la pandemia del COVID-19, tomando en consideración la interacción con los usuarios de esta red social, para medir por una parte el nivel de engagement de los usuarios con cada una de las marcas y, por otra parte, las opiniones vertidas a través de los comentarios efectuados en cada una de las publicaciones durante la emergencia sanitaria.

\section{Materiales y métodos}

El presente estudio es de carácter descriptivoexplicativo con enfoque mixto, que parte de un mapeo georreferenciado de los hoteles categoría lujo de la ciudad de Manta mediante el uso de la plataforma ArcGIS, para contextualizar el estudio desde la perspectiva geográfica. Para la evaluación del uso de la red social Facebook por parte de los hoteles se aplicó el modelo PRGS (Presencia, Respuesta, Generación y Sugerencia) diseñado por el Bureau Interactivo de Publicidad de España (IAB por sus siglas en inglés) en el año 2013 y utilizado en estudios científicos posteriores (Ávila Campoverde y Ugalde, 2020; Beltrán-Bueno et al., 2017; Sánchez Jiménez, 2018; Sánchez Casado y Giraldo Cardona, 2015). Las cuatro dimensiones del modelo y sus implicaciones en este estudio se describen según el siguiente detalle:

- $\quad$ Presencia: analiza el número de seguidores y el número de publicaciones realizadas por parte de la página de Facebook de la marca (hotel).

- Respuesta: para el caso del presente estudio, se refiere a la suma total de reacciones manifestadas a través de "me gusta, me encanta, me im- porta, me divierte, me asombra, me entristece, y me enoja", que tienen las publicaciones.

- Generación: hace referencia al número de comentarios realizados por parte de los seguidores en las publicaciones.

- Sugerencia: es el número de publicaciones compartidas por parte de los seguidores a otros usuarios de la red social Facebook.

Cada una de las dimensiones del modelo PRGS fueron obtenidos por medio de las cuentas oficiales de los hoteles a partir de la declaratoria del primer caso de contagio del virus COVID-19 en el Ecuador (29 de febrero del 2020) hasta el 21 de agosto del 2020 (fecha de corte de este estudio), sumando un total de 174 días.

Basado en la metodología de Ávila Campoverde y Ugalde (2020) y Sánchez Jiménez (2018), para la recolección de datos se utilizó la plataforma online de pago Fanpage Karma, lo que permitió monitorear cuantitativamente cada una de las variables PRGS, las mismas que nos sirvieron de insumo en la obtención del engagement (nivel de compromiso) de los seguidores para con los hoteles.

Para el cálculo del engagement de los seguidores de los hoteles categoría lujo de Manta en la red social Facebook, se utilizó una fórmula basada en Mejía Llano (2013) y Castelló Martínez (2013) como se citó en Ávila Campoverde y Ugalde (2020), la cual se aplica mediante la sumatoria del número de reacciones, más los comentarios emitidos, más las publicaciones compartidas por los seguidores, éstas se dividen para el número de publicaciones hechas por la marca y éste por el número de seguidores multiplicado por 100 (Ecuación 1).

engagement $=\frac{\text { Número de reacciones }+ \text { Número de comentarios }+ \text { Número de publicaciones compartidas }}{\text { Número de seguidores }} \times 100$

Finalmente, los comentarios realizados por los seguidores de las páginas de los hoteles durante la pandemia fueron recopilados y procesados mediante el programa Atlas.Ti versión 9, para efectuar el análisis cualitativo de los datos, a fin de conocer las principales opiniones de los seguidores de los hoteles categoría lujo de Manta.

\section{Resultados y discusión}

Manta es uno de los 22 cantones de la provincia costera de Manabí en Ecuador. Su población es de 226.477 habitantes (Instituto Nacional de Estadística y Censos [INEC], 2010). Se sitúa al suroeste de la provincia de Manabí, a orillas del océano Pacífico, 
con una extensión de $306 \mathrm{~km}^{2}$, limita al norte y oeste con el océano Pacífico, al este con los cantones Jaramijó y Montecristi, y al sur con el cantón Montecristi; además, posee conexión aérea, terrestre y marítima, se compone de dos parroquias rurales, y cinco urbanas, estas últimas concentran el 96,06 \% de la población del cantón (Gobierno Autónomo Descentralizado del Cantón Manta, 2014) entre las que destaca la ciudad de Manta, que históricamente ha tenido un rol predominante en el dinamismo económico de la provincia (Baque Cantos et al., 2018) y agrupa una gran variedad hotelera (Mora Pisco et al., 2019).

Los hoteles con categoría lujo de la ciudad de Manta, fueron determinados mediante el cruce de in- formación oficial, científica y comercial. Se encontró que existe disonancia en la información almacenada en los registros del Ministerio de Turismo del Ecuador (MINTUR), en relación a la categorización de los hoteles. En este contexto, de acuerdo al catastro de MINTUR (versión actualizada 2020), el hotel Wyndham Sail Plaza no aparece en el registro y la categorización difiere a la denominación presentada en agencias virtuales de viaje (OTA por sus siglas en inglés), como Trip Advisor, Booking.com y Trivago, y estudios previos realizados en relación a esta categoría de establecimientos de alojamiento en el destino (Rangel Anchundia, 2019), por lo que se le dio relevancia a la información científica y comercial. El resultado de esta categoría se detalla en la Tabla 1.

Tabla 1. Hoteles categoría de lujo de Manta en Facebook

\begin{tabular}{lccc}
\hline \multicolumn{1}{c}{ Hotel } & Categoría & $\begin{array}{c}\text { Seguidores } \\
\text { (al corte del estudio) }\end{array}$ & Dirección web en Facebook \\
\hline Hotel Poseidón & Lujo & 56.109 & facebook.com/poseidonmanta \\
Hotel Oro Verde & Lujo & 38.350 & facebook.com/HotelOroVerdeManta \\
Hotel Mantahost & Lujo & 25.332 & facebook.com/Mantahosthotel \\
Hotel Wyndham Sail Plaza & Lujo & 21.056 & facebook.com/WyndhamMantaSailPlaza \\
Hotel Balandra & Lujo & 18.575 & facebook.com/hotelbalandra \\
\hline
\end{tabular}

Como se observa en la Tabla 1, la mayoría de los hoteles no poseen una cantidad superior a 30.000 seguidores, exceptuando a los hoteles Poseidón y Oro Verde que superan esta cifra. Esto evidencia que actualmente los establecimientos de alojamiento de esta categoría en el destino Manta, no están aprovechando el enorme potencial de la red social Facebook para difundir información relevante sobre su marca, pese a estar ubicados estratégicamente en el destino, a lo largo de la zona de playa del cantón Manta (Figura 1).

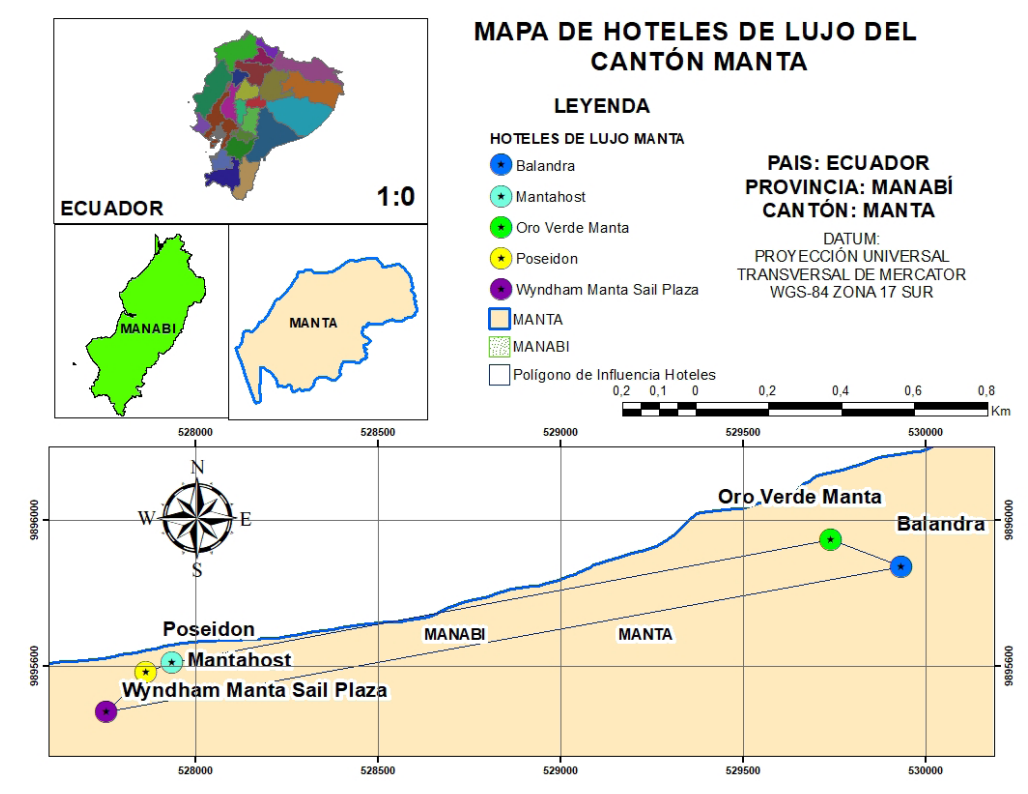

Figura 1. Georreferenciación de hoteles categoría de lujo de Manta. 
Como se observa en la Figura 1, los hoteles Mantahost, Wyndham Sail Plaza y Poseidón se agrupan en un radio aproximado de 180,75 metros conformando un complejo de hoteles frente a la playa de Barbasquillo, de la cual se encuentran a una distancia promedio de 94,31 metros. En el caso de los hoteles Oro Verde y Balandra se encuentran agrupados en un radio estimado de 380,3 metros frente a la playa El Murciélago (la más concurrida de Manta), a una distancia (de la playa) de 126,47 y 329,31 metros, respectivamente.

\subsection{Medición del engagement de los hoteles en Facebook}

\subsubsection{Presencia}

En la medición de la presencia, se identificaron dos grupos de hoteles con categoría de lujo de la ciudad de Manta, en relación con la presencia en la red social Facebook. El primer grupo está conformado por los hoteles con un número de seguidores mayor a 30.000. Aquí se observó que Poseidón es la marca con el mayor número de fans pese a representar el menor número de publicaciones durante el confinamiento, representando aproximadamente un $46 \%$ más seguidores que el hotel Oro Verde que ocupa el segundo lugar en este orden. En el segundo grupo se encuentran las marcas en las que se detectó menos de 26.000 seguidores conformado por los hoteles Mantahost, Wyndham Sail Plaza y Balandra, siendo este último el de menor número, por debajo de 20.000. Respecto al número de publicaciones, el hotel Mantahost presenta la mayor cantidad de publicaciones durante el periodo de confinamiento, con un total de 162 contenidos subidos en su muro, lo cual representa un $50 \%$ más publicaciones que el hotel Oro Verde (el segundo con más publicaciones). Lo anterior, justifica que el hotel Mantahost sea el establecimiento de alojamiento con más contenido en su muro por día presentando una tasa de 0,931 publicaciones diarias, que evidencian su compromiso e intención de comunicación constante con los seguidores de su página (Tabla 2).

Tabla 2. Presencia de los hoteles en Facebook (1 de marzo - 21 de agosto)

\begin{tabular}{lccccc}
\hline \multicolumn{1}{c}{ Facebook } & Mantahost & Poseidón & Oro Verde & Wyndham Sail Plaza & Balandra \\
\hline Número de fans & 25.332 & 56.109 & 38.350 & 21.056 & 18.575 \\
Número de publicaciones & 162 & 71 & 108 & 77 & 87 \\
Número de publicaciones por día & 0,931 & 0,408 & 0,620 & 0,442 & 0,5 \\
\hline
\end{tabular}

\subsubsection{Respuesta}

El hotel Poseidón es el establecimiento con mayor número de respuestas (me gusta, me encanta, me importa, me divierte, me asombra, me entristece, me enoja) a sus publicaciones, las cuales alcanzan un total de 5.983 reacciones, $41 \%$ más que el hotel Oro Verde (4.235) el cual ocupa el segundo lugar. Se puede afirmar que existe una relación directa entre el número de respuestas con su número de fans, y se encontró que, a mayor número de fans, mayor número de reacciones presentan las publicaciones (Tabla 3).

Tabla 3. Respuestas acumuladas en los seis meses en Facebook

\begin{tabular}{lc}
\hline \multicolumn{1}{c}{ Hoteles } & Facebook \\
\hline Poseidón & 5.983 \\
Oro Verde & 4.235 \\
Mantahost & 2.330 \\
Wyndham Sail Plaza & 1.729 \\
Balandra & 1.621 \\
\hline
\end{tabular}

El hotel Oro Verde presenta el porcentaje más elevado en respuestas a sus publicaciones en rela- ción con el número de seguidores con un 11,04\% seguido del hotel Mantahost con un porcentaje del 
$10,66 \%$. Esta ratio muestra la calidad de la publicación presentada por los hoteles en su página de Face- book y cuánto de esa información es de interés de los seguidores en dicha red social (Tabla 4).

Tabla 4. Porcentaje de respuestas en relación con el número de seguidores

\begin{tabular}{lc}
\hline \multicolumn{1}{c}{ Hoteles } & Facebook \\
\hline Oro Verde & $11,04 \%$ \\
Poseidón & $10,66 \%$ \\
Mantahost & $9,2 \%$ \\
Balandra & $8,72 \%$ \\
Wyndham Sail Plaza & $8,21 \%$ \\
\hline
\end{tabular}

A partir del comienzo del estado de excepción oficial, y por consiguiente el confinamiento obligatorio en el territorio ecuatoriano ( 16 de marzo del 2020), se evidencia la disminución de la interacción de los seguidores de los hoteles con las publicaciones que se posteaban en el muro de Facebook, a pesar de que éstas continuaban a un menor ritmo, en comparación con el período antes de la emergencia sanitaria. La falta de respuesta hacia las publicaciones se refleja en mayor grado con el hotel Poseidón, que, desde el 14 de marzo hasta el 23 de abril de 2020 , presentó un crecimiento en reacciones de un
$1,15 \%$ (considerando el tiempo de 6 meses para el estudio). A su vez, se evidencia que el primer hotel en proponer estrategias, en reacción a lo sucedido tras la declaración de emergencia es el hotel Wyndham Sail Plaza (28 de marzo) experimentando un crecimiento acentuado en el número de respuestas de sus seguidores a sus publicaciones en contraste con los primeros días de ese mes, luego el hotel Oro Verde (13 de abril) para continuar con el Mantahost (21 de abril), seguido del Poseidón (22 de abril) y finalmente a principios de mayo se observa la reacción del hotel Balandra (Figura 2).

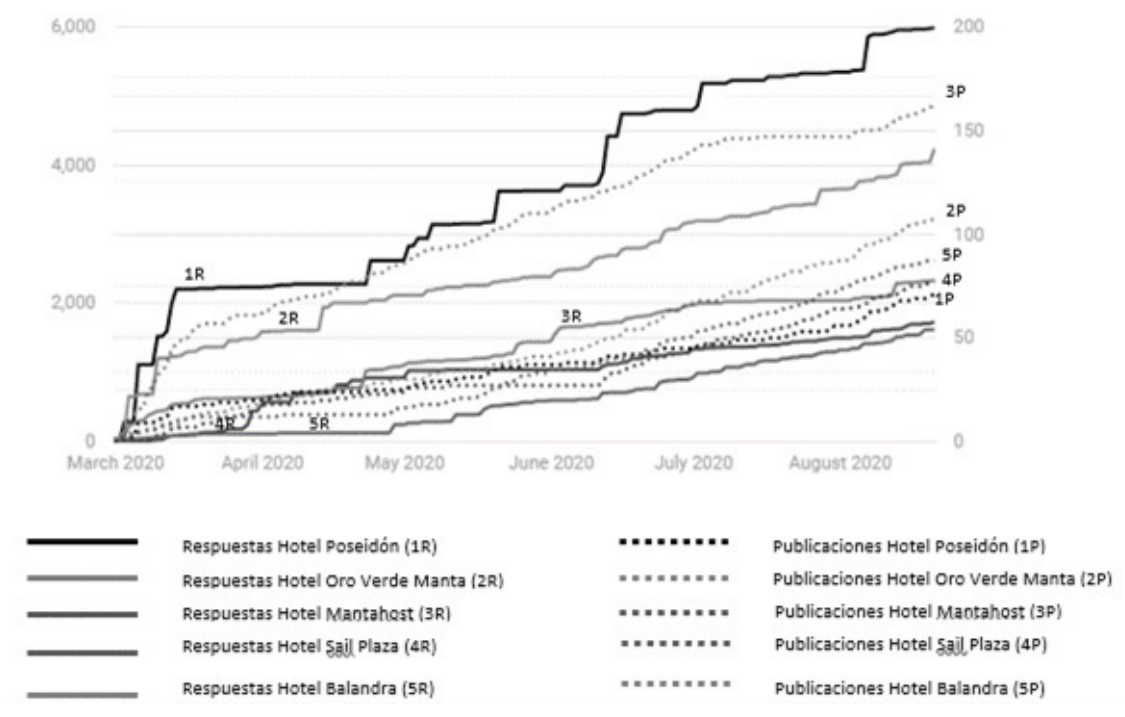

Figura 2. Número de Respuestas vs. Número de Publicaciones. Elaborado a partir de Fanpage Karma.

\subsubsection{Generación}

El hotel Poseidón presenta el número más alto en generación que evidencian sus seguidores en las publicaciones de Facebook con 324 comentarios, esto representa un $27 \%$ más aportaciones de sus seguidores que el segundo en este orden, ocupado por el hotel Oro Verde, con 255 comentarios (Tabla 5). 
Tabla 5. Número de comentarios

\begin{tabular}{lc}
\hline \multicolumn{1}{c}{ Hoteles } & Facebook \\
\hline Poseidón & 324 \\
Oro Verde & 255 \\
Mantahost & 219 \\
Balandra & 157 \\
Wyndham Sail Plaza & 59 \\
Elaborado a partir de Fanpage Karma. & \\
\hline
\end{tabular}

En el análisis de la variable generación (Figura 3 ), se observa que cada uno de los hoteles tuvieron diferentes períodos para ejercer la presión hacia sus seguidores en la realización de comentarios de sus publicaciones, de los cuales, el hotel Poseidón fue el último en reaccionar (21 de mayo) a diferencia de los hoteles como el Wyndham Sail Plaza (29 de marzo) y
Oro Verde (14 de abril). El hotel Balandra presenta una reacción leve a partir de los primeros días de mayo, con un crecimiento más intencional en los primeros días de julio. Finalmente, el hotel Mantahost a diferencia del resto de hoteles evidencia un crecimiento lento, aunque constante en la generación de comentarios de sus seguidores durante el período de la emergencia.

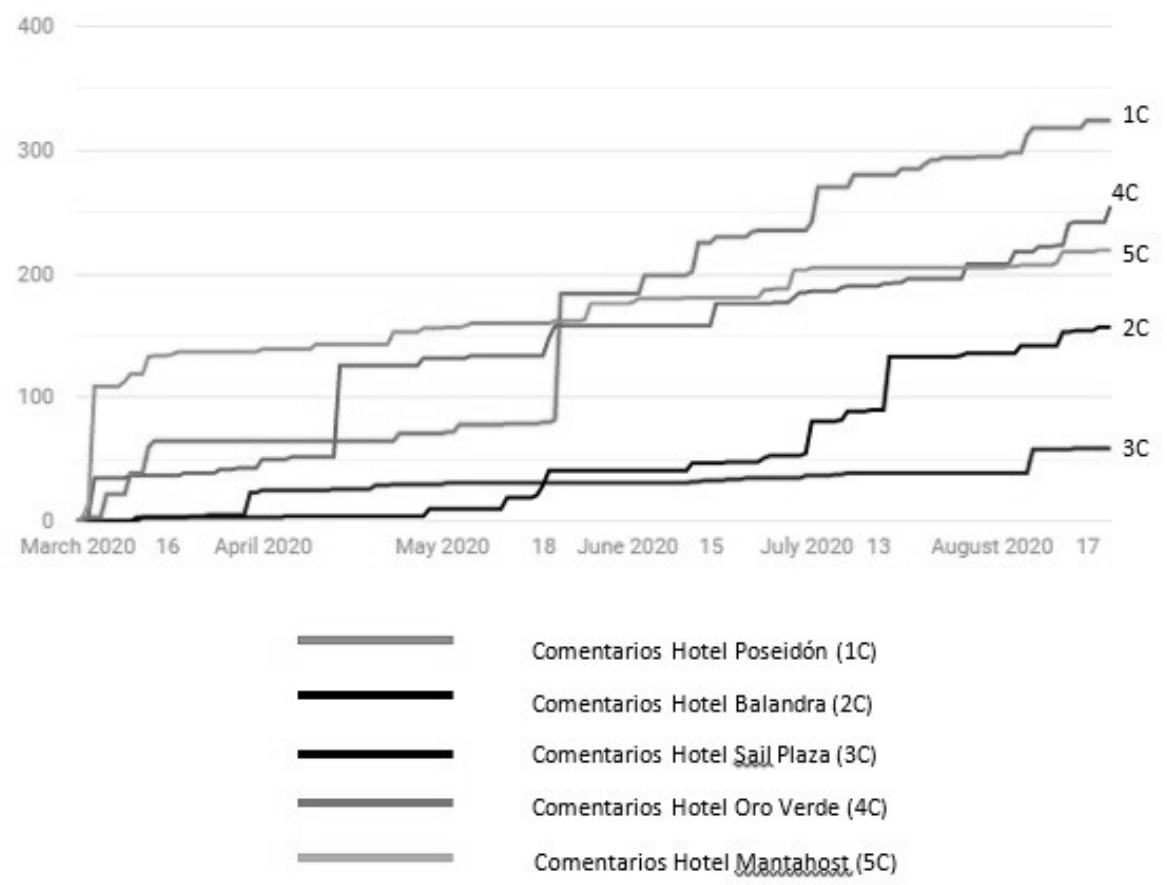

Figura 3. Número de comentarios en las publicaciones. Elaborado a partir de Fanpage Karma

\subsubsection{Sugerencias}

El hotel Oro Verde posee el mayor número de sugerencias (compartidos) en sus publicaciones durante el período de emergencia (717), seguido muy de cerca por el hotel Balandra (712), esto representa alrededor del $20 \%$ más que el hotel Poseidón (590 comparticiones) a pesar del mayor número de publicaciones y número de fans que posee este último establecimiento (Tabla 6). Ante esto se observa que el número de seguidores y la cantidad de publicaciones no condicionan la variable Sugerencia (publicaciones compartidas). 
Tabla 6. Número de sugerencias (compartidos)

\begin{tabular}{cc}
\hline Hoteles & Facebook \\
\hline Oro Verde & 717 \\
Balandra & 712 \\
Poseidón & 590 \\
Mantahost & 485 \\
Wyndham Sail Plaza & 340 \\
\hline
\end{tabular}

Elaborado a partir de Fanpage Karma.

A diferencia de los gráficos expuestos por Respuesta y Generación, en Sugerencia no se evidencian saltos en crecimientos considerables que indiquen el interés de los seguidores por compartir las publicaciones, evidenciando la ausencia de contenido atractivo posteado por parte de la marca (Figura 4).

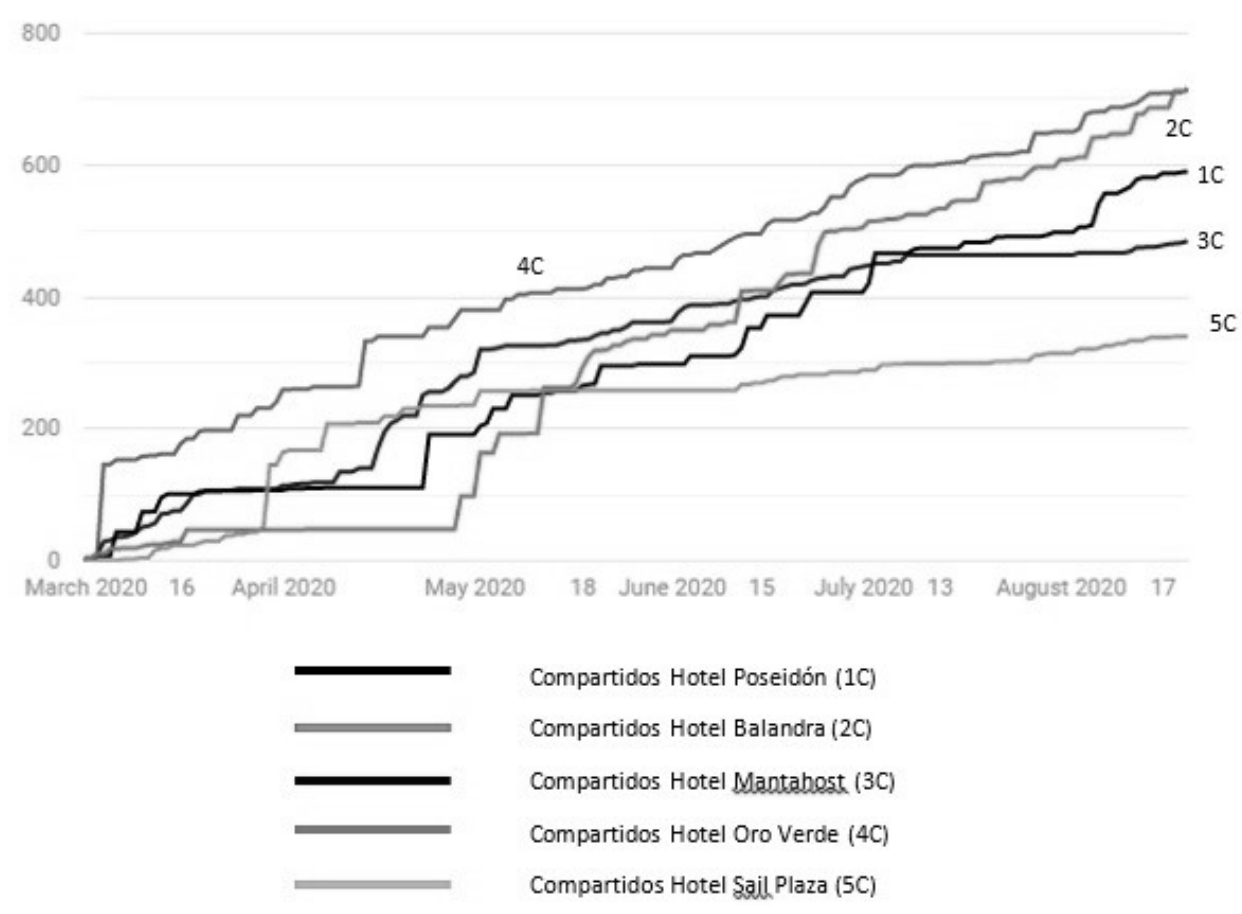

Figura 4. Número de sugerencias (compartidos). Elaborado a partir de Fanpage Karma

\subsubsection{Engagement}

Según Leander, como se citó en Buhalis y Mamalakis (2015), el promedio de engagement en páginas de Facebook que tienen más de 10.000 fans es $0,96 \%$. Al tomar como referencia lo anterior, Leander sostiene que un engagement por encima del $1 \%$ es bueno, entre el $0,5 \%$ y $0,99 \%$ son valores considerados dentro del promedio mientras que niveles de compromiso por debajo del $0,5 \%$ se puede afirmar que los contenidos promovidos en la red social por parte de la marca no alcanzan las expectativas de los seguidores.

Como se observa en la Tabla 7, y según los resultados del engagement de los hoteles de categoría lujo de Manta, ninguno de los establecimientos alcanza el $0,5 \%$ para ingresar dentro de los valores promedio. Por el contrario, el hotel que más se acerca a dicho rango es el Poseidón con 0,17\%, seguido del Balandra $0,15 \%$, Wyndham Sail Plaza 0,13\%, Oro Verde 0,12 $\%$, y finalmente Mantahost con $0,07 \%$. 
Tabla 7. Resultados del engagement

\begin{tabular}{lc}
\hline \multicolumn{1}{c}{ Hoteles } & Facebook \\
\hline Poseidón & $0,17 \%$ \\
Balandra & $0.15 \%$ \\
Wyndham Sail Plaza & $0,13 \%$ \\
Oro Verde & $0,12 \%$ \\
Mantahost & $0,07 \%$ \\
\hline
\end{tabular}

Elaborado a partir de Fanpage Karma

Del resultado obtenido, se logró determinar que, en la mayoría de los casos, existió apatía de los seguidores hacia las publicaciones suscitadas durante la emergencia sanitaria. De la misma manera, los hoteles también experimentaron fuerte incertidumbre ante los escenarios ocasionados por la pandemia. Otro aspecto a tomar en cuenta, fue la poca importancia que los hoteles le brindaron a la comunicación por medio de sus páginas de Facebook durante el confinamiento, evidenciando reacciones tardías en la actualización de contenido relevante para atraer la atención de sus seguidores.

\subsection{Contenido de los comentarios en publicaciones de Facebook}

El análisis cualitativo mediante el programa informático Atlas. Ti v.9 determinó nueve segmentos de interés en los comentarios enviados, los cuales fueron: concurso; en vivo; etiquetado e interacción entre usuarios; oferta de ventas; opinión sobre hotel y sus servicios; opinión de la ciudad; saludos, apoyo y agradecimiento; solicitud de información y requerimiento de servicios; vinculados directamente a la pandemia.

La estrategia de organización de un concurso a través de Facebook, fue realizada únicamente por el hotel Mantahost, generando un total de 61 comentarios ( $42 \%$ del total de mensajes), que se convirtió en el indicador por el que más mensajes obtuvo dicho establecimiento.

La realización de en vivo, fue organizada solo por el hotel Wyndham Sail Plaza obteniendo 71 mensajes en reacción de sus publicaciones (31\% del total) y se convirtió el motivo de la mayoría de sus comentarios. Además, a partir de este evento se originaron comentarios en opinión de la ciudad, resaltando las bondades de su paisaje urbano y natural con 5 respuestas de ese tipo.
El $18 \%$ del total de los comentarios efectuados hacia las páginas de Facebook de los hoteles, fueron realizados con base en el indicador etiquetado e interacción entre usuarios, es decir, etiquetar a una o varias personas en reacción a una publicación para que esa o esas personas puedan verla. La página del hotel Poseidón, fue la que tuvo mayor número de publicaciones, que dieron cabida a 45 mensajes de este tipo.

La oferta de servicios en los comentarios (venta de alimentos, ofrecimientos para ser proveedor del hotel, etc.) por parte de los seguidores es una reacción poco común. En efecto, el hotel Oro Verde es el único que posee este tipo de reacciones por parte de sus fans con 5 comentarios.

Una buena opinión sobre el hotel y sus servicios lleva a que más personas confíen en las publicaciones que se promueven, en este código, el hotel Wyndham Sail Plaza se convirtió en el que más comentarios a favor de este indicador originó (54 respuestas). El $16 \%$ del total de comentarios (114) hacia los hoteles, son promovidos sobre la base de este tipo de mensajes.

Los comentarios de saludos, apoyo y agradecimiento hacia los hoteles, generaron un total de 70 respuestas, en los que el hotel Wyndham Sail Plaza resultó como el que más reacciones originó con 42 comentarios.

La solicitud de información y requerimiento de servicio es el indicador más importante a tomar en cuenta dentro de los comentarios de las publicaciones, dado que, a través del mismo, se observan las intenciones de los seguidores para adquirir o comprar un producto o servicio. Además, es el motivo por el que más comentarios se generaron hacia los hoteles (222) que representan un $28 \%$ del total de 
respuestas. Dentro de este indicador el hotel que más comentarios generó fue el hotel Poseidón (107), abarcando un $57 \%$ del total de respuestas relacionadas con este motivo. A partir de ello, se deduce que el hotel Poseidón fue el que tuvo más oportunidad de monetizar sus servicios a través de Facebook.
Por último, existieron comentarios vinculados directamente con la pandemia (preguntas sobre la próxima apertura, mensajes de ánimo a las personas que han sufrido por el virus, añoranzas por volver a las instalaciones de los hoteles, etc.). Se generaron 49 respuestas de este tipo (Tabla 8).

Tabla 8. Vínculo de comentarios hacia los hoteles

\begin{tabular}{|c|c|c|c|c|c|}
\hline \multirow[b]{2}{*}{ Códigos } & \multicolumn{4}{|c|}{ Hoteles } & \multirow[b]{2}{*}{ Totales } \\
\hline & $\begin{array}{l}\text { Mantahost } \\
\text { Gr }=136\end{array}$ & $\begin{array}{c}\text { Poseidón } \\
\mathrm{Gr}=175\end{array}$ & $\begin{array}{c}\text { Sail Plaza } \\
\text { Gr }=120\end{array}$ & $\begin{array}{l}\text { Oro Verde } \\
\text { Manta } \\
\text { Gr }=108\end{array}$ & \\
\hline $\begin{array}{l}\text { Concurso } \\
\mathrm{Gr}=61\end{array}$ & 61 & 0 & 0 & 0 & 61 \\
\hline $\begin{array}{l}\text { En vivo } \\
\mathrm{Gr}=71\end{array}$ & 0 & 0 & 71 & 0 & 71 \\
\hline $\begin{array}{l}\text { Etiquetado e interacción entre usu- } \\
\text { arios } \\
\mathrm{Gr}=117\end{array}$ & 34 & 45 & 21 & 17 & 117 \\
\hline $\begin{array}{l}\text { Oferta de ventas } \\
\text { Gr }=5\end{array}$ & 0 & 0 & 0 & 5 & 5 \\
\hline $\begin{array}{l}\text { Opinión sobre el hotel y su servicio } \\
\text { Gr=114 }\end{array}$ & 13 & 23 & 54 & 24 & 114 \\
\hline $\begin{array}{l}\text { Opinión sobre la ciudad } \\
\mathrm{Gr}=5\end{array}$ & 0 & 0 & 5 & 0 & 5 \\
\hline $\begin{array}{l}\text { Saludos, apoyo y agradecimiento } \\
\mathrm{Gr}=70\end{array}$ & 7 & 4 & 42 & 17 & 70 \\
\hline $\begin{array}{l}\text { Solicitud Información y requer- } \\
\text { imiento de servicio } \\
\mathrm{Gr}=186\end{array}$ & 22 & 107 & 7 & 50 & 186 \\
\hline $\begin{array}{l}\text { Vinculado directamente a la pan- } \\
\text { demia } \\
\mathrm{Gr}=43\end{array}$ & 7 & 9 & 22 & 5 & 43 \\
\hline Totales & 144 & 188 & 222 & 118 & 672 \\
\hline
\end{tabular}

Como se observa en la Tabla 8 , los hoteles presentaron mayores comentarios bajo los códigos relacionados a solicitud de información y requerimiento de servicio (222), opinión sobre el hotel y su servicio (143) y etiquetado e interacción entre usuarios (142), los cuales se enmarcan en la promoción de los hoteles por medio de la red social Facebook en medio de la pandemia.

\section{Conclusiones}

La georreferenciación de los hoteles bajo estudio determinó que todos están ubicados estraté- gicamente frente a la línea de playa, en un radio de tres kilómetros. Los hoteles Wyndham Sail Plaza, Mantahost y Poseidón agrupados geográficamente, conforman un complejo de hoteles asentados frente a la zona de playa de Barbasquillo, en un radio de 180,75 metros, mientras que los hoteles Oro Verde y Balandra, se agrupan en un radio menor a 380,03 metros frente a la playa El Murciélago (la más concurrida de Manta). Por esta razón, se afirma que, en ninguno de los casos, la localización de los establecimientos estudiados representa una ventaja comparativa entre ellos, que pueda incidir en cuanto al número de seguidores en sus páginas de Facebook. 
Al corte de esta investigación, se determinó que los hoteles con mayor número de seguidores en esta red social son Poseidón con 56.638, seguido por el hotel Oro Verde con 39.436, mientras que los de menor número fueron Mantahost con 26.067, Wyndham Sail Plaza con 22.389, y Balandra con 18.575. La aplicación del modelo PRGS, demostró independencia en el resultado de sus variables, donde los valores encontrados en cada una de ellas, no está condicionado por el número de seguidores que contienen sus páginas. De los datos obtenidos a través de la plataforma Fanpage Karma, se concluye que los hoteles presentaron ausencias prolongadas y baja actividad en Facebook durante el tiempo de la emergencia sanitaria, provocando un bajo nivel de interacción entre la marca y sus fans.

La medición del engagement, demostró que los hoteles bajo estudio tuvieron un débil manejo de la red social Facebook para la promoción turística en tiempos de COVID-19, proporcionando valores inferiores a $0,5 \%$. El engagement sobre $1 \%$ se considera bueno, mientras valores de $0,5 \%$ a $0,99 \%$ se consideran aceptables en páginas con más de 10.000 seguidores (Leander, como se citó en Buhalis y Mamalakis, 2015). Poseidón obtuvo el valor más alto $(0,17 \%)$ seguido por Balandra $(0,15 \%)$, sin embargo, ambos porcentajes están lejos del promedio mínimo aceptable, mientras que Mantahost, pese a ser la marca con mayor cantidad de contenido publicado (162) posee el promedio más bajo de engagement $(0,07 \%)$, esto demuestra que el nivel de compromiso (engagement) no

\section{Referencias}

Alcalá Casillas, M. (2017). Internet, instrumento esencial para el ejercicio del derecho a la información. Derecom, 23, 117-127.

Altamirano, D. (2015). Repercusión del uso de Facebook relacionado al destino turístico Trujillo en los visitantes sociales. Universidad Nacional de Trujillo.

Avila Campoverde, F., \& Ugalde, C. (2020). Instagram: la red social con la mayor interacción para promover los destinos turísticos ecuatorianos. Investigaciones Turísticas, O(19), 50-72. https://doi.org/10.14198/INTURI2020.19.03

Baque Cantos, M. A., Baque Sánchez, E. R., Chiquito Tigua, G. P., \& Baque Parrales, S. M. (2018). se condiciona por el número de seguidores, sino por la capacidad que tenga la marca para crear contenidos relevantes para los usuarios de la red.

En el análisis cualitativo mediante el programa Atlas.Ti 9, de los comentarios realizados (c) por los seguidores de las páginas de los hoteles categoría de lujo de Manta, se determinaron 9 códigos en relación a concurso (61c); en vivo (71c); etiquetado e interacción entre usuarios (142c); oferta de ventas $(5 \mathrm{c})$; opinión sobre hotel y sus servicios (143c); opinión de la ciudad (6c); saludos, apoyo y agradecimiento (81c); solicitud de información y requerimiento de servicios (222c); y vinculados a la pandemia (49c). De acuerdo con este estudio, el hotel Poseidón tuvo más oportunidades de monetizar sus servicios, presentando el mayor número de comentarios $(107 \mathrm{c})$ con relación al código solicitud de información y requerimiento de servicios. Por el contrario, el hotel Wyndham Sail Plaza, aunque generó el mayor número total de comentarios (222c) por encima de los otros hoteles, no supusieron un verdadero interés por la adquisición de servicios, debido a que la mayoría se identificaron bajo el código en vivo, y solo siete bajo solicitud de información y requerimiento de servicios. En este sentido, se infiere que los concursos promovidos a través de Facebook no generan un incremento de engagement con la marca, por lo tanto, los hoteles deben crear contenidos que generen interés en la solicitud de información para promover sus ventas.

Microempresas en el Ecuador: Caso ciudad de Manta. Dominio de las Ciencias, 4(1), 619632. https://doi.org/10.23857/dc.v4i1.771

Beltrán-Bueno, M., Parra-Meroño, M. \& Padi1la-Piernas, J. (2017). Las redes sociales aplicadas al sector hotelero. International Journal of Scientific Management and Tourism, 3(2), 131-154.

Buhalis D., \& Mamalakis E. (2015) Social Media Return on Investment and Performance Evaluation in the Hotel Industry Context. In I, Tussyadiah, A. Inversini (eds), Information and Communication Technologies in Tourism 2015 (pp. 241-253). Springer. https://doi. org/10.1007/978-3-319-14343-9_18 
Burgueño, E. (2020). Países con el mayor número de usuarios de Facebook en América Latina en abril de 2020. https:/es.statista.com/estadisticas/1173450/paises-mayor-cantidad-usuarios-facebook-america-latina/

Chierichetti, L. (2012). La promoción del turismo musical: los festivales de música en Facebook. PASOS. Revista de Turismo y Patrimonio Cultural, 10(4), 49-58. https://doi.org/10.25145/j. pasos.2012.10.052

Duarte, F. (9 de septiembre de 2019). Los países en los que la gente pasa más tiempo en las redes sociales (y los líderes en América Latina). $B B C$ Mundo. https://www.bbc.com/mundo/noticias-49634612https://www.bbc.com/mundo/ noticias-49634612

El Comercio. (14 de julio de 2020). 630 hoteles del Ecuador permanecen cerrados; algunos administradores analizan la suspensión definitiva. $E l$ Comercio. https://www.elcomercio.com/actualidad/hoteles-ecuador-cerrados-pandemia-coronavirus.html

El Universo. (6 de julio de 2020). Con solo el $9 \%$ de ocupación, hoteles ya consideran perdidos el 2020 y el 2021. El Universo. https://www.eluniverso.com/noticias/2020/07/03/nota/7894014/ hoteleria-turismo-impacto-covid-19-desempleo-iliquidez-cierre

Félix, Á. \& García Reinoso, N. (2020). Estudio de pérdidas y estrategias de reactivación para el sector turístico por crisis sanitaria COVID-19 en el destino Manta-Ecuador. Revista Internacional de Turismo, Empresa y Territorio (RITUREM), 4(1), 79-103. https://doi.org/10.21071/riturem.v4i1.12743

Félix, Á., García Reinoso, N. \& Vera, R. (2020). Participatory diagnosis of the tourism sector in managing the crisis caused by the pandemic (COVID-19). Revista Interamericana de Ambiente y Turismo, 16(1), 66-78. https://doi. org/10.4067/s0718-235x2020000100066

Gobierno Autónomo Descentralizado del Cantón Manta. (2014). Plan de Desarrollo y Ordenamiento Territorial del Cantón Manta 2014-2019. Gobierno Autónomo Descentralizado del Cantón Manta.

Gössling, S., Scott, D., \& Hall, C. M. (2020). Pandemics, tourism and global change: a rapid as- sessment of COVID-19. Journal of Sustainable Tourism, 29(1), 1-20. https://doi.org/10.1080/0 9669582.2020.1758708

Hsu, Y. L. (2012). Facebook as international e Marketing strategy of Taiwan hotels. International Journal of Hospitality Management, 31(3), 7298. https://doi.org/10.1016/j.ijhm.2011.11.005

Instituto Nacional de Estadística y Censos [INEC]. (2010). Población y Demografía http://www. ecuadorencifras.gob.ec/censo-de-poblacion-y-vivienda/

Leung, X. Y. (2013). The marketing effectiveness of hotel Facebook pages: from perspectives of customers and messages. University of Nevada, Las Vegas.

López-Pérez, L., \& Olvera-Lobo, M.-D. (2016). Comunicación pública de la ciencia a través de la web 2.0. El caso de los centros de investigación y universidades públicas de España. Profesional De La Información, 25(3), 441-448. https:// doi.org/10.3145/epi.2016.may.14

Mellinas, J. P., Martínez María-Dolores, S. M. \& Bernal García, J. (2014). Uso de las redes sociales en los hoteles de la costa española. Investigaciones Turísticas, 0(7), 87-101. https://doi. org/10.14198/INTURI2014.7.05

Miranda Bonilla, H. (2016). El acceso a Internet como derecho fundamental. Revista Jurídica IUS Doctrina, 9(15), 1-23.

Mora Pisco, C. I., Montilla Pacheco, A. J. \& Mora Pisco, L. L. (2019). Perspectivas y potencialidades del cantón Manta para la implementación de un plan de marketing turístico. TURyDES, 12(26), https://www.eumed.net/rev/turydes/26/ marketing-turistico-manta.html

Morán Quiñonez, C. J., \& Cañarte-Rodríguez, T. C. (2020). Las redes sociales aplicadas en los hoteles en el destino turístico del Sector Crucita - Manabí. Revista Cientifica FIPCAEC (Fomento de la investigación y publicación en Ciencias Administrativas, Económicas y Contables), 5(5), 64-81. https://doi.org/10.23857/ fipcaec.v5i5.216

Ortíz Garcìa, L. \& González Sánchez, R. (2014). La social media como herramienta de mejora de 
la experiencia turística: una aplicación al sector hotelero. Revista Iberoamericana de Turismo RITUR, 4(1), 16-34.

Parra-Meroño, M. C., Beltrán-Bueno, M. A., \& Padilla-Piernas, J. M. (2017). El fenómeno web 2.0 aplicado al sector hotelero. International Journal of Scientific Management and Tourism, 3(4), 301-320.

Rangel Anchundia, L. (2019). Análisis de la reputación online de los Hoteles de Lujo de la ciudad de Manta. Revista San Gregorio, 1(33). http:// dx.doi.org/10.36097/rsan.v1i33.883

Sánchez Amboage, E. (2010). Promoción de un destino turístico a través de las redes sociales: el caso de A Coruña en Facebook. Redmarka. Revista de Marketing Aplicado, 2(5), 11-52. https://doi.org/10.17979/redma.2010.01.05.4712
Sánchez Casado, N. \& Giraldo Cardona, C. M. (2015). Análisis de la actividad en redes sociales de marcas del sector moda como herramienta de social commerce. Anuario de Jóvenes Investigadores, $8,72-74$.

Sánchez Jiménez, M. A. (2018). Análisis de la estrategia en las redes sociales oficiales desarrollada por el Consejo de Promoción Turística de México. Revista CIMEXUS, 13(1), 13-29. https:// doi.org/10.33110/cimexus13101

Zazueta Hernández, M. M., \& Olmos Martínez, E. (2018). El uso de Facebook y Twitter como herramienta de mercadotecnia en la industria de la hospitalidad en México. En M. Cabrera Méndez y R. Díez Somavilla (dirs.), Análisis y tendencias de las redes sociales. VIII edición del Congreso Internacional sobre Redes Sociales Comunica2 (pp. 98-116). Universitat Politècnica de València. 